\title{
The time has come for physicians to take notice: the impact of psychosocial stressors on the heart.
}

Vincent M. Figueredo, M.D.

Thomas Jefferson University

Follow this and additional works at: https://jdc.jefferson.edu/cardiologyfp

Part of the Cardiology Commons

Let us know how access to this document benefits you

\section{Recommended Citation}

Figueredo, M.D., Vincent M., "The time has come for physicians to take notice: the impact of psychosocial stressors on the heart." (2009). Division of Cardiology Faculty Papers. Paper 4. https://jdc.jefferson.edu/cardiologyfp/4

This Article is brought to you for free and open access by the Jefferson Digital Commons. The Jefferson Digital Commons is a service of Thomas Jefferson University's Center for Teaching and Learning (CTL). The Commons is a showcase for Jefferson books and journals, peer-reviewed scholarly publications, unique historical collections from the University archives, and teaching tools. The Jefferson Digital Commons allows researchers and interested readers anywhere in the world to learn about and keep up to date with Jefferson scholarship. This article has been accepted for inclusion in Division of Cardiology Faculty Papers by an authorized administrator of the Jefferson Digital Commons. For more information, please contact: JeffersonDigitalCommons@jefferson.edu. 
As submitted to: American Journal of Medicine

and later published as:

American Journal of Medicine

Volume 122, Issue 8, August 2009, Pages 704-712

DOI: 10.1016/j.amjmed.2009.05.001

\title{
THE TIME HAS COME FOR PHYSICIANS TO TAKE NOTICE: THE IMPACT OF PSYCHOSOCIAL STRESSORS ON THE HEART
}

\author{
Vincent M. Figueredo, MD FACC FAHA FASE \\ Einstein Institute for Heart and Vascular Health, Albert Einstein Medical Center, and Jefferson \\ Medical College, Philadelphia, PA
}

Running head: Psychosocial Stressors and Cardiovascular Disease

Key words: stress, depression, psychosocial stressor, cardiovascular, coronary artery disease

Disclosures: no conflict of interest

Address correspondence to: Vincent M. Figueredo, MD

Einstein Institute for Heart and Vascular Health 
5501 Old York Road, Levy 3

Philadelphia, PA 19141

TEL: $215-456-8819$

FAX: 215-456-3533

E-mail: figueredov@einstein.edu

\section{$\underline{\text { ABSTRACT }}$}

A rapidly growing body of evidence supports a relationship between psychosocial factors and cardiovascular disease. In this paper, a review of the epidemiological and clinical research investigating this relationship concludes that psychosocial stressors can be both a cause and consequence of cardiovascular disease events. Furthermore, recent data has shown that stress management may reduce future cardiac events in cardiovascular disease patients. Unfortunately, the influence of psychosocial risk factors on cardiovascular disease remains under-recognized compared to traditional cardiac risk factors. Physicians and their associates should screen for psychosocial stressors and recognize potential symptoms. Consideration should be given to developing improved liaison relationships with psychological or behavioral specialists to facilitate more specialized interventions when appropriate. A variety of interventions conducted by appropriately trained mental health professionals have successfully improved stress in patients with cardiovascular disease and other chronic diseases. The time has come for physicians to recognize the impact of psychosocial stressors on cardiovascular disease. 
A growing body of evidence supports a relationship between psychosocial stressors and chronic disease progression. The influence of psychosocial stressors on chronic illnesses remains under recognized, when compared to other risk factors. Chronic disease states, which may be impacted by psychosocial factors, include cardiovascular disease, diabetes, cancer, rheumatoid arthritis, human immunodeficiency virus, and chronic obstructive pulmonary disease. Psychological factors, which have the potential to affect the onset or progression of chronic diseases, include depression, anxiety, anger/hostility, acute and chronic life stressors, and lack of social support.

The purpose of this paper is to 1 . review epidemiological and clinical data investigating the relationship between psychosocial factors and cardiovascular disease, 2. review data suggesting stress management may reduce future cardiac events, and 3. conclude the time has come for physicians recognize the impact of psychosocial factors on cardiovascular disease.

\section{Chronic Psychosocial Factors and Cardiovascular Disease}

Data exploring the relationship between psychosocial factors and cardiovascular disease come primarily from epidemiological studies and prospective studies examining laboratory responses to acute stressors with long term follow up of subjects for events. The Harvard Mastery of Stress Study examined the reliability of severe anxiety expressed in laboratory testing as a marker for susceptibility of future cardiovascular disease and other chronic illnesses. ${ }^{1}$ College students underwent stress experiments, including mental arithmetic with harassment by experimenter, and sonic confusion; hearing one's own voice delayed 0.2 seconds while trying to retell a story from memory as accurately and as rapid as possible under threat of electric shock. After 35 years, the 
frequency of severe anxiety during testing in college was 59\% in those who had developed cardiovascular disease, compared to $27 \%$ in healthy subjects. Of note, at time of publication, there were six deaths, five of whom had experienced severe anxiety in response to stress experiments carried out in college.

INTERHEART studied the association of psychosocial risk factors with risk of acute myocardial infarction in 11,119 cases and 13,648 controls from 52 countries. $^{2}$ In this case control study, psychosocial stress was assessed by questions assessing stress at work and home, financial stress, major life events in the past year, and the presence of depression. Moderate or severe general stress (work, home, or both) had an OR=1.65 for acute myocardial infarction (adjusted for geographical region, age, sex, and smoking). This increased risk was consistent across regions, ethnic groups, and gender. Permanent general stress had an $\mathrm{OR}=2.17$ for acute myocardial infarction; severe financial stress 1.33, stressful life events 1.48, and depression 1.55.

A meta-analysis of 11 prospective studies of asymptomatic, healthy subjects by Rugulies et al., found the presence of clinical depression was associated with major adverse cardiac events $\mathrm{RR}=2.69) .{ }^{3}$ As a comparison, in the Framingham Heart Study, presence of hypertension had a $\mathrm{RR}=1.92$ for major adverse cardiac events. ${ }^{4}$ In the Normative Aging Study, 735 men over 60 years old without coronary artery disease were assessed for anxiety characteristics. ${ }^{5}$ After a 12.4 years, there were 11 myocardial infarctions in the lowest anxiety quartile compared to 29 in the highest quartile. The adjusted $\mathrm{RR}=1.43$ for myocardial infarction associated with each standard deviation increase in overall anxiety. 
The impact of low social support, hostility and anger on progression of coronary atherosclerosis was studied in 137 subjects with coronary artery disease on baseline coronary angiogram. ${ }^{6}$ Patients completed self-report questionnaires concerning social support, anger and hostility. After two years, subjects with low social support and high anger expression had an $\mathrm{OR}=30$ for coronary artery disease progression relative to subjects reporting high social support and low anger.

Changes in left ventricular ejection fraction as a function of mental stress-testing were examined in 126 coronary artery disease patients subjected to mental tasks during radionuclide ventriculography. ${ }^{7}$ Those who evidenced mental stress-induced ischemia with a decrease in left ventricular ejection fraction were less likely to remain cardiovascular disease event free over five-years (Figure 1). Lesperance et al., studied long-term survival after myocardial infarction in relation to the Beck Depression Inventory Score measured during myocardial infarction hospitalization. $^{8}$ Depression symptoms during myocardial infarction admission were closely linked to long-term survival.

\section{Work Stress and Cardiovascular Disease}

Chronic work stress, defined as high psychological demands and low decision latitude, was associated with cardiovascular disease events $(\mathrm{RR}=1.68)$ in 10,308 middle aged London-based civil servants. ${ }^{9}$ Associations were found between work stress and negative behaviors such as low physical activity and poor diet, and pathophysiological consequences such as decreased 
heart rate variability and higher morning rise in a.m. cortisol. The authors concluded work stress may be an important determinant of cardiovascular disease among working age population, mediated through indirect effects on health behaviors and direct effects on neuroendocrine pathways.

Recurrent cardiovascular disease events among 972 myocardial infarction patients returning to work were examined in a prospective cohort study. ${ }^{10}$ High job strain (high psychological demands and low decision latitude) was an independent predictor of recurrent cardiovascular disease in a multivariate model adjusted for 26 potentially confounding factors $(\mathrm{HR}=2.00)$.

\section{Marital Stress and Cardiovascular Disease}

A longitudinal analysis of 192 couples over 17 years in Tecumseh, Michigan placed couples into categories: both partners communicate their anger, one spouse expresses while the other suppresses, and both husband and wife suppress their anger and brood. ${ }^{11}$ In 26 pairs where both suppressed their anger, there were 13 deaths: in $27 \%$ of couples one partner died and in $23 \%$ both died. In the remaining 166 pairs (one or both spouses communicate anger), there were 41 deaths: in $19 \%$ of couples one partner died and in $6 \%$ both died.

In the Healthy Women Study, 393 women completed a seven item measure of relationship

quality. ${ }^{12}$ B-mode carotid ultrasound measured plaque burden after 11 years. Greater plaque burden was found in those who reported low satisfaction with marital quality compared to 
satisfied wives (Figure 2). Unmarried women were intermediate in plaque burden. Three years later, a subset who reported low marital satisfaction had significant progression to moderate to severe levels of plaque burden compared to satisfied women. The authors conclude that high quality marriages may protect against cardiovascular disease. Further study will hopefully confirm these findings.

\section{$\underline{\text { Acute Stress and Cardiovascular Disease }}$}

Subjects from the Multicenter Investigation of the Limitation of Infarct Size (MILIS) study were questioned about possible triggers for their myocardial infarction. ${ }^{13}$ Of 849 subjects, $49 \%$ reported a possible trigger; the most common being emotional upset, followed by physical activity, lack of sleep, and overeating.

The Northridge earthquake struck Los Angeles January 17, 1994. The Los Angeles County Coroner reported a sharp increase in cardiovascular disease -related sudden deaths on the day of the earthquake. ${ }^{14}$ During the week prior, sudden death occurred 4.6 times per day. On the day of the earthquake there were 24 sudden cardiac deaths.

Two hundred New York area implantable cardioverter-defibrillator patients had their stored electrocardiograms reviewed for ventricular tachyarrhythmias triggering implantable cardioverter-defibrillator therapy before and after the World Trade Center attack of September 
11, 2001..$^{15}$ There was a 2.3 fold increase in ventricular tachyarrhythmias during the month following 9/11, relative to other months between May 2001 and October 2002.

An interesting example of the effect of acute emotional stress on the heart is tako-tsubo or transient left ventricular apical ballooning syndrome. Patients present after acute emotional distress with symptoms and electrocardiographic changes consistent with acute myocardial. Left ventriculogram reveals apical ballooning and a hypercontractile basal segment, a shape reminiscent of the pot (tsubo) that Japanese fishermen use to catch octopus (tako). Recent data implicate massive catecholamine release causing stress-induced myocardial stunning. In a study of 22 tako-tsubo patients, ${ }^{16}$ psychological stressors included death of relative, domestic abuse, arguments, catastrophic medical diagnosis, devastating financial or gambling losses.

In addition to severe emotional upset, catastrophic events have been found to trigger myocardial infarction; earthquakes, missile attacks, and...you're team losing a World Cup football game in a penalty shootout. Carroll et al., studied myocardial infarction admissions in London after England lost to Argentina in a penalty shootout during the 1998 World Cup. ${ }^{17}$ On the day of the match, there was a $25 \%$ increase in myocardial infarction admissions compared to the same day in prior years. Increased admissions persisted for two days after England's loss, returning to baseline by day three.

Similarly, in a study examining daily cardiovascular disease events in Munich during the 2006 World Cup, cardiovascular emergencies increased 2.7 times in men and 1.8 times in women on the days in which Germany was participating in the quarterfinals and semifinals. ${ }^{18}$ On these 
days, myocardial infarction and major arrhythmia admissions increased 3 times compared to the same day in previous years (Figure 3). The highest incidence of cardiac events occurred during the first two hours of the games.

Pathophysiological Mechanisms of Psychosocial Factors and Cardiovascular Disease

\section{Pathogenesis}

Behavioral mechanisms: Psychosocial stressors contribute to a higher frequency of adverse health behaviors; smoking, alcohol bingeing and abuse, poor dietary compliance, physical inactivity, and poor adherence to medical regimens. Further, psychosocial risk factors and negative behaviors frequently cluster, such as job strain, depression and poor dietary compliance. This clustering is similar to that seen with more traditional risk factors such as diabetes and dyslipidemia. $^{19}$

Pathophysiological mechanisms of chronic stress: In addition to promoting unhealthy behaviors, chronic stressors activate the sympathetic nervous system. ${ }^{20}$ Repeated sympathetic stimulation increases heart rate and blood pressure. ${ }^{20-22}$ Resulting decreased heart rate variability ${ }^{9}$ and baroreflex dysfunction ${ }^{23}$ due to autonomic nervous system dysfunction have been associated with increased cardiovascular disease events. Depression increases levels of inflammatory markers including fibrinogen, C-reactive protein, interleukin-6, and tumor necrosis factor. ${ }^{24-27}$ 
Chronic stressors also activate the hypothalamic-pituitary-adrenal axis producing hypercortisolemia. ${ }^{9} \quad$ Hypercortisolemia promotes central obesity and insulin resistance, risk factors for cardiovascular disease.

However, Whooley et al., question the importance of pathophysiological mechanisms mediating the association between depression and cardiovascular disease. ${ }^{28}$ In a study of 1017 stable coronary artery disease patients, the age-adjusted rate of cardiovascular events was $10 \%$ among patients with a Patient Health Questionnaire (see American Heart Association Advisory below) score greater than nine and $6.7 \%$ among participants without depressive symptoms. $\mathrm{HR}=1.31$ was reduced to a non-significant 1.05 after adjusting for potential behavioral mediators, including physical inactivity, medication non-adherence, and tobacco use.

Pathophysiological mechanisms of acute stress: Acute stress activates the sympathetic nervous system. Studies of acute stress testing have demonstrated: increases in heart rate and blood pressure, coronary vasoconstriction, and decreased myocardial electrical stability. ${ }^{19}$

\section{European Guidelines on Cardiovascular Disease Prevention and Clinical Practice}

The Fourth Joint Task Force of the European Society of Cardiology on Cardiovascular Disease Prevention and Clinical Practice concluded, "there is increasing scientific evidence that psychosocial factors contribute independently to the risk of coronary heart disease, even after statistical control for the effects of standard risk factors." 29 Further, "in addition to increasing the risk of a first event and worsening the prognosis of cardiovascular disease, these factors may act 
as barriers to treatment adherence and efforts to improve lifestyle." From a mechanistic standpoint, psychosocial risk factors, not only portend to risky health behaviors, but also result in pathophysiological characteristics such as autonomic, endocrine, and inflammatory changes involved in promoting cardiovascular disease.

2008 American Heart Association Science Advisory on Depression and Coronary Heart

\section{$\underline{\text { Disease }}$}

Endorsed by the American Psychiatric Association, an American Heart Association Scientific Advisory was recently published, entitled, "Depression and Coronary Heart Disease: Recommendations for Screening, Referral, and Treatment." ${ }^{30}$ This multispecialty consensus document reports as many as $20 \%$ of myocardial infarction patients meet criteria for major depression, and an even greater number demonstrate elevated depressive symptom levels. Recognizing that, beyond the pathophysiological effects on the heart, depression is associated with decreased adherence to medications and injurious behaviors, the Advisory proposes an algorithm and Patient Health Questionnaires (PHQ) healthcare practitioners can use in cardiovascular disease patients to determine whether depression is present (Figure 4 and Tables 1 and 2). They recommend starting with PHQ-2 (Table 1). If the patient answers yes to either question, then continue with PHQ-9 (Table 2). Questions are scored: not at all=0; several days $=1$; more than half the days $=2$; and nearly every day $=3$; then added together to get a depression severity score. The Advisory stresses that although there is currently no direct evidence that depression screening leads to improved outcomes in cardiovascular disease patients, depression is linked with increased cardiovascular disease morbidity and mortality, 
poorer risk factor modification, and lower medication and cardiac rehabilitation compliance. Treatment options are proposed including antidepressant pharmacotherapy, cognitive behavioral therapy, and cardiac rehabilitation. The Advisory stresses that patients with positive screening should be evaluated by a mental health professional. Hence, coordination of care between healthcare providers is essential in patients with combined medical and mental health diagnoses.

\section{Psychosocial and Behavioral Intervention Trials}

A recent meta-analysis, reporting on psychological treatment of cardiac patients identified 43 randomized trials; 23 which reported mortality data for 9,856 patients. ${ }^{31}$ When comparing psychological treatment plus usual care to usual care alone, the $\mathrm{OR}=0.72$ for all cause mortality at two years. Mortality benefits only applied to men. However, only 10 studies allowed gender analysis. Psychological treatment was statistically superior to control for reduction of heart rate and total cholesterol. Additionally, psychological treatment was superior to control in improving both social support and quality of life.

In the Myocardial Ischemia Intervention Trial, 107 coronary artery disease patients with ischemia during mental stress testing or ambulatory electrocardiographic monitoring were

randomized to a four month program of exercise or stress management. ${ }^{32}$ Patients living a distance from the facility formed a nonrandom, usual care comparison group. Exercise training consisted of aerobic exercise three times per week for 16 weeks. Stress management included sixteen 1.5 hour cognitive behavioral therapy group sessions conducted by a clinical psychologist. Sessions included education, cognitive and behavioral skills training, progressive 
muscle relaxation, and at least two individual biofeedback sessions. Stress management was associated with a lower risk of adverse cardiac events at five years compared with usual care $(\mathrm{RR}=0.26$; Figure 5). Exercise was associated with a non-significant lowering of cardiac event risk $(R R=0.68)$.

Blumenthal et al., followed this study with a randomized control trial comparing aerobic exercise and stress management training with routine medical care in 134 stable coronary artery disease patients with exercise-induced ischemia. ${ }^{33}$ Patients in the exercise and stress management groups versus usual care had less depression (Beck Depression Inventory), and reduced distress (General Health Questionnaire). Exercise and stress management groups had smaller reductions in left ventricular ejection fraction during mental stress testing versus usual care. In a subset of patients with significant stress-induced wall motion abnormalities at baseline, exercise and stress management groups had lower wall motion abnormality rating scores and greater improvements in brachial flow-mediated dilation versus usual care. In a subgroup study, patients receiving stress management had improved baroreflex sensitivity and increases in heart rate variability. The authors concluded for stable coronary artery disease patients, exercise and stress management training reduced emotional distress and improved cardiovascular risk markers more than usual medical care.

Milani and Lavie compared 522 coronary artery disease patients enrolled in a cardiac rehabilitation program 2000 to 2005 to 179 patients who did not complete rehabilitation. ${ }^{34}$ Outpatient cardiac rehabilitation consisted of 12 weeks of 36 educational and exercise sessions. The prevalence of depressive symptoms (Kellner Symptom Questionnaire) decreased 63\% 
following rehabilitation (17 to 6\%). Depressed patients following rehabilitation had a significant increased mortality at 3.5 years versus non-depressed patients (22 versus 5\%). Interestingly, depressed patients who completed rehabilitation had a 73\% lower mortality compared with control-depressed subjects who did not complete rehabilitation. A reduction in depressive symptoms was related to improvement in fitness as evidenced by improved peak oxygen consumption during exercise.

Not all studies have demonstrated a benefit of cognitive behavioral therapy after myocardial infarction. In the Enhancing Recovery in Coronary Heart Disease Patients (ENRICHD) trial, 2,481 post-myocardial infarction patients were randomized to cognitive behavioral therapy or usual medical care. ${ }^{35}$ Cognitive behavioral therapy was initiated at a median of 17 days after myocardial infarction for six months, plus group therapy when feasible, and selective serotonin reuptake inhibitors for patients scoring higher than 24 on the Hamilton Rating Scale for Depression (HRSD). The main outcome measure was a composite endpoint of death or recurrent myocardial infarction. Secondary outcomes included change in HRSD or Enhancing Recovery in Coronary Heart Disease Patients Social Support Instrument (ESSI) scores. Cognitive behavioral therapy did not increase event free survival. It did improve depression and social isolation scores. Of note, the relative improvement in the psychological intervention group compared with the usual care group was less than expected due to substantial improvement in the usual care patients. A nonrandomized, post-hoc analysis of ENRICHD found that patients treated with an SSRI, whether assigned to receive cognitive behavioral therapy or usual care, had a $42 \%$ reduction in death or recurrent myocardial infarction compared with depressed patients not receiving an $\mathrm{SSRI}^{36}$ 
The Sertraline AntiDepressant Heart Attack Trial (SADHART) investigation demonstrated that sertraline is safe and efficacious in depressed patients with ischemic heart disease but was underpowered to detect a mortality difference between sertraline and placebo. ${ }^{37}$

Therefore, while encouraging data suggest intervention can impact coronary artery disease patients with psychosocial stressors, further study will be required to determine which treatments definitively provide benefit. Additionally, future studies prospectively assessing therapies for specific stressors and their ability to decrease events offer the best chance to further elucidate potential pathophysiological versus behavioral mechanisms underlying this relationship.

\section{The Time Has Come For Physicians to Take Notice!}

The influence of psychosocial risk factors on cardiovascular disease remains under recognized compared to traditional cardiac risk factors. Does this mean physicians should act as mental health professionals? No. But given the strong relationship between psychosocial risk factors and cardiovascular disease physicians need to be proactive participants in their patients' care. Because physicians are accustomed to obtaining medical histories, additional questions about psychosocial risk factors can be incorporated in the review of systems.

The recent American Heart Association Science Advisory on Depression in Coronary Heart Disease, recommends screening for depression in cardiovascular disease patients. ${ }^{30}$ Their proposed algorithm (Figure 4) offers a strategy for assessment and referral of cardiovascular 
disease patients with depressive symptoms. PHQ-2, and PHQ-9 if needed (Tables 1 and 2), are brief questionnaires for assessing depressive symptoms in cardiovascular disease patients that can be used during office visits. The Executive Summary of the European Guidelines on Cardiovascular Disease Prevention and Clinical Practice offers tips to help physicians work with their cardiovascular disease patients to assess potential psychosocial stressors in their life (Table 3). ${ }^{29}$ One new approach to fostering this more compassionate, identifying relationship between patient and healthcare practitioner is Narrative Medicine, which seeks to improve effectiveness of care by developing the capacity for attention, reflection, representation, and affiliation with patients and colleagues. ${ }^{38}$ The time has come for physicians to take notice. Encouraging data suggest intervention can impact coronary artery disease patients with psychosocial stressors. Further study will be required to determine which treatments definitively provide benefit. Importantly, in addition to increasing the risk of cardiovascular events and worsening the prognosis of cardiovascular disease, psychosocial factors may act as barriers to treatment adherence and efforts to improve lifestyle. 


\section{REFERENCES}

1. Russek LG, King SH, Russek SJ, Russek HI. The Harvard Mastery of Stress Study 35year follow-up: prognostic significance of patterns of psychophysiological arousal and adaptation. Psychosom Med 1990;52(3):271-85.

2. Rosengren A, Hawken S, Ounpuu S, Sliwa K, Zubaid M, Almahmeed WA, et al. Association of psychosocial risk factors with risk of acute myocardial infarction in 11119 cases and 13648 controls from 52 countries (the INTERHEART study): case-control study. Lancet 2004;364(9438):953-62.

3. Rugulies R. Depression as a predictor for coronary heart disease. a review and metaanalysis. Am J Prev Med 2002;23(1):51-61.

4. Wilson PW, D'Agostino RB, Levy D, Belanger AM, Silbershatz H, Kannel WB. Prediction of coronary heart disease using risk factor categories. Circulation 1998;97(18):1837-47.

5. Lesperance F, Frasure-Smith N, Talajic M, Bourassa MG. Five-year risk of cardiac mortality in relation to initial severity and one-year changes in depression symptoms after myocardial infarction. Circulation 2002;105(9):1049-53.

6. Angerer P, Siebert U, Kothny W, Muhlbauer D, Mudra H, von Schacky C. Impact of social support, cynical hostility and anger expression on progression of coronary atherosclerosis. J Am Coll Cardiol 2000;36(6):1781-8.

7. Shen BJ, Avivi YE, Todaro JF, Spiro A, 3rd, Laurenceau JP, Ward KD, et al. Anxiety characteristics independently and prospectively predict myocardial infarction in men the 
unique contribution of anxiety among psychologic factors. J Am Coll Cardiol 2008;51(2):113-9.

8. Jiang W, Babyak M, Krantz DS, Waugh RA, Coleman RE, Hanson MM, et al. Mental stress--induced myocardial ischemia and cardiac events. JAMA 1996;275(21):1651-6.

9. Chandola T, Britton A, Brunner E, Hemingway H, Malik M, Kumari M, et al. Work stress and coronary heart disease: what are the mechanisms? Eur Heart J 2008;29(5):6408.

10. Aboa-Eboule C, Brisson C, Maunsell E, Masse B, Bourbonnais R, Vezina M, et al. Job strain and risk of acute recurrent coronary heart disease events. JAMA 2007;298(14):1652-60.

11. Harburg E, Julius M, Kaciroti N, Gleiberman L, Schork MA. Expressive/suppressive anger-coping responses, gender, and types of mortality: a 17-year follow-up (Tecumseh, Michigan, 1971-1988). Psychosom Med 2003;65(4):588-97.

12. Gallo LC, Troxel WM, Kuller LH, Sutton-Tyrrell K, Edmundowicz D, Matthews KA. Marital status, marital quality, and atherosclerotic burden in postmenopausal women. Psychosom Med 2003;65(6):952-62.

13. Tofler GH, Stone PH, Maclure M, Edelman E, Davis VG, Robertson T, et al. Analysis of possible triggers of acute myocardial infarction (the MILIS study). Am J Cardiol 1990;66(1):22-7.

14. Leor J, Poole WK, Kloner RA. Sudden cardiac death triggered by an earthquake. N Engl J Med 1996;334(7):413-9. 
15. Steinberg JS, Arshad A, Kowalski M, Kukar A, Suma V, Vloka M, et al. Increased incidence of life-threatening ventricular arrhythmias in implantable defibrillator patients after the World Trade Center attack. J Am Coll Cardiol 2004;44(6):1261-4.

16. Sharkey SW, Lesser JR, Zenovich AG, Maron MS, Lindberg J, Longe TF, et al. Acute and reversible cardiomyopathy provoked by stress in women from the United States. Circulation 2005;111(4):472-9.

17. Carroll D, Ebrahim S, Tilling K, Macleod J, Smith GD. Admissions for myocardial infarction and World Cup football: database survey. BMJ 2002;325(7378):1439-42.

18. Wilbert-Lampen U, Leistner D, Greven S, Pohl T, Sper S, Volker C, et al. Cardiovascular events during World Cup soccer. N Engl J Med 2008;358(5):475-83.

19. Rozanski A, Blumenthal JA, Kaplan J. Impact of psychological factors on the pathogenesis of cardiovascular disease and implications for therapy. Circulation 1999;99(16):2192-217.

20. Carney RM, Freedland KE, Veith RC. Depression, the autonomic nervous system, and coronary heart disease. Psychosom Med 2005;67 Suppl 1:S29-33.

21. Blumenthal JA, Thyrum ET, Siegel WC. Contribution of job strain, job status and marital status to laboratory and ambulatory blood pressure in patients with mild hypertension. $\mathrm{J}$ Psychosom Res 1995;39(2):133-44.

22. Steptoe A, Cropley M, Joekes K. Job strain, blood pressure and response to uncontrollable stress. J Hypertens 1999;17(2):193-200.

23. Thomas KS, Nelesen RA, Ziegler MG, Bardwell WA, Dimsdale JE. Job strain, ethnicity, and sympathetic nervous system activity. Hypertension 2004;44(6):891-6. 
24. Steptoe A, Kunz-Ebrecht S, Owen N, Feldman PJ, Rumley A, Lowe GD, et al. Influence of socioeconomic status and job control on plasma fibrinogen responses to acute mental stress. Psychosom Med 2003;65(1):137-44.

25. Ranjit N, Diez-Roux AV, Shea S, Cushman M, Seeman T, Jackson SA, et al. Psychosocial factors and inflammation in the multi-ethnic study of atherosclerosis. Arch Intern Med 2007;167(2):174-81.

26. Vaccarino V, Johnson BD, Sheps DS, Reis SE, Kelsey SF, Bittner V, et al. Depression, inflammation, and incident cardiovascular disease in women with suspected coronary ischemia: the National Heart, Lung, and Blood Institute-sponsored WISE study. J Am Coll Cardiol 2007;50(21):2044-50.

27. Anisman H, Merali Z. Cytokines, stress, and depressive illness. Brain Behav Immun 2002;16(5):513-24.

28. Whooley MA, de Jonge P, Vittinghoff E, Otte C, Moos R, Carney RM, et al. Depressive symptoms, health behaviors, and risk of cardiovascular events in patients with coronary heart disease. JAMA 2008;300(20):2379-88.

29. Graham I, Atar D, Borch-Johnsen K, Boysen G, Burell G, Cifkova R, et al. European guidelines on cardiovascular disease prevention in clinical practice: executive summary. Eur Heart J 2007;28(19):2375-414.

30. Lichtman JH, Bigger JT, Jr., Blumenthal JA, Frasure-Smith N, Kaufmann PG, Lesperance F, et al. Depression and Coronary Heart Disease. Recommendations for Screening, Referral, and Treatment. A Science Advisory From the American Heart Association Prevention Committee of the Council on Cardiovascular Nursing, Council on 
Clinical Cardiology, Council on Epidemiology and Prevention, and Interdisciplinary Council on Quality of Care and Outcomes Research. Circulation 2008.

31. Linden W, Phillips MJ, Leclerc J. Psychological treatment of cardiac patients: a metaanalysis. Eur Heart J 2007;28(24):2972-84.

32. Blumenthal JA, Jiang W, Babyak MA, Krantz DS, Frid DJ, Coleman RE, et al. Stress management and exercise training in cardiac patients with myocardial ischemia. Effects on prognosis and evaluation of mechanisms. Arch Intern Med 1997;157(19):2213-23.

33. Blumenthal JA, Sherwood A, Babyak MA, Watkins LL, Waugh R, Georgiades A, et al. Effects of exercise and stress management training on markers of cardiovascular risk in patients with ischemic heart disease: a randomized controlled trial. JAMA 2005;293(13):1626-34.

34. Milani RV, Lavie CJ. Impact of cardiac rehabilitation on depression and its associated mortality. Am J Med 2007;120(9):799-806.

35. Berkman LF, Blumenthal J, Burg M, Carney RM, Catellier D, Cowan MJ, et al. Effects of treating depression and low perceived social support on clinical events after myocardial infarction: the Enhancing Recovery in Coronary Heart Disease Patients (ENRICHD) Randomized Trial. JAMA 2003;289(23):3106-16.

36. Taylor CB, Youngblood ME, Catellier D, Veith RC, Carney RM, Burg MM, et al. Effects of antidepressant medication on morbidity and mortality in depressed patients after myocardial infarction. Arch Gen Psychiatry 2005;62(7):792-8.

37. Glassman AH, O'Connor CM, Califf RM, Swedberg K, Schwartz P, Bigger JT, Jr., et al. Sertraline treatment of major depression in patients with acute MI or unstable angina. JAMA 2002;288(6):701-9. 
38. Charon R. The patient-physician relationship. Narrative medicine: a model for empathy, reflection, profession, and trust. JAMA 2001;286(15):1897-902. 


\section{Figure Legends}

1. Probability of cardiovascular event-free survival as a function of mental stress-induced left ventricular ejection fraction $(\mathrm{EF})$ changes. Reprinted with permission from Jiang et al., JAMA $1996 ; 275: 1651-1656 .^{8}$

2. A: Percent of each marital status/quality group with a plaque score of two or higher at the first scan $(\mathrm{N}=376)$. B: Percent of each group showing an increase in plaque score from 0 or 12 to 2 or higher. * Groups differ significantly at $\mathrm{p}<.05$. Reprinted with permission from Gallo et al., Psychosomatic Medicine 2003;65:952-962. ${ }^{12}$

3. Daily cardiovascular events in the study population from May 1 to July 31 in 2003, 2005, in 2006. The FIFA World Cup 2006 in Germany started on June 9, 2006, and ended on July 9, 2006. The 2006 World Cup matches with German participation are indicated by the numbers 1 through 7: match 1, Germany verse Costa Rica; match 2, Germany versus Poland; match 3, Germany versus Ecuador; match 4, Germany versus Sweden; match 5, Germany versus Argentina; match 6, Germany versus Italy; and match 7, Germany versus Portugal (for thirdplace standing). Match 8 was the final match, Italy versus France. Reprinted with permission from Wilbert-Lampen et al., N Engl J Med 2008;358:475-483. ${ }^{18}$

4. American Heart Association Science Advisory on Depression and Coronary Heart Disease algorithm screening for depression in cardiovascular disease patients. *Meets diagnostic criteria for major depression, has a PHQ-9 score of 10-19, has had no more than 1 or 2 prior episodes of 
depression, and screens negative for bipolar disorder, suicidality, significant substance abuse, or other major psychiatric problems. $\uparrow$ Meets the diagnostic criteria for major depression and 1) has a PHQ-9 score $\geq 20$; or 2) has had 3 or more prior depressive episodes; or 3) screens positive for bipolar disorder, suicidality, significant substance abuse, or other major psychiatric problem. \$If "Yes" to Q.9 "suicidal," immediately evaluate for acute suicidality. If safe, refer for more comprehensive clinical evaluation; if at risk for suicide, escort the patient to the emergency department. Reprinted with permission from Lichtman JH et al., Circulation 2008; Sep 29. [Epub ahead of print $]^{30}$.

5. Cumulative time to cardiovascular event curves for exercise, stress management, and usual care groups. After adjusting for age, baseline left ventricular ejection fraction, and history of myocardial infarction, stress management was associated with a significantly lower risk (relative risk, 0.26; $\mathrm{P}=.04$ ) of an adverse cardiac event compared with usual care. Exercise was also associated with a lower relative risk compared with usual care, but this difference was not statistically significant (relative risk, $0.68 ; \mathrm{P}=.34$ ). * Indicates significantly different from usual care at $\mathrm{P}<.05$. Reprinted with permission from Blumenthal et al., Arch Intern Med $1997 ; 157: 2213-2223 .^{32}$ 